\title{
Les méthodes et les limites de la dosimétrie après contamination interne
}

\author{
E. BLANCHARDON ${ }^{1}$, A. FLÜRY-HERARD ${ }^{2}$, F. PAQUET $^{3}$
}

(Manuscrit reçu le 24 avril 2007, accepté le 17 juillet 2007)

RÉSUMÉ L'estimation des doses absorbée, équivalente et efficace résultant d'une contamination interne se base sur la mesure de l'activité par anthroporadiamétrie (in vivo) ou par analyse radiotoxicologique in vitro. La valeur d'activité mesurée est interprétée en termes de dose à l'aide de modèles biocinétiques et dosimétriques qui décrivent le devenir des radionucléides dans le corps d'un individu de référence et permettent de calculer l'énergie qu'ils y déposent. Pour s'assurer du respect des limites réglementaires de dose, des programmes de surveillance sont mis en place. Les mesures d'activité peuvent être interprétées suivant une procédure standard comme celle proposée par le groupe IDEAS. Cependant, une incertitude significative est introduite dans le calcul de la dose par les incertitudes de mesure, par la méconnaissance partielle des conditions d'exposition et par le réalisme imparfait des modèles. En plus de cette incertitude, l'extrapolation aux faibles doses d'effets biologiques observés après une irradiation plus intense, la toxicité chimique des radionucléides et l'hétérogénéité du dépôt d'énergie rendent difficile l'estimation du risque résultant d'une contamination interne. En conclusion, il convient de garder à l'esprit que la dose efficace est un outil de gestion de la radioprotection mais pas un indicateur quantitatif du risque individuel.

ABSTRACT The methods and limitations of dosimetry after internal contamination.

The assessment of absorbed, equivalent and effective doses after internal exposure is based on activity measurement by in vivo counting or bioassay. A dose value is derived from this measurement by means of biokinetic and dosimetric models which describe the behaviour of radionuclides in the body of a reference man and the subsequent deposition of energy. To enforce regulatory dose limits, monitoring programs are designed. The doses can be estimated from the measurement data according to a standard procedure as proposed by the IDEAS group. However, a significant uncertainty is associated with the dose estimate, due to the uncertainties of measurement, to the incomplete knowledge of the conditions of exposure and to the imperfect realism of the models. In addition to this uncertainty, the extrapolation to low dose of biological effects observed at higher dose, the chemical toxicity of radionuclides and the heterogeneous energy deposition make the estimation of the risk following an internal exposure very difficult. As a conclusion, it should be reminded that the effective dose is a tool for the management of radiological protection but not a quantitative indicator of the individual risk.

Keywords: internal dosimetry / dosimetry / internal exposure / dose calculation / contamination

\footnotetext{
IRSN, Laboratoire d'Évaluation de la Dose Interne, DRPH/SDI/LEDI, B.P. 17, 92262 Fontenay-aux-Roses, France.

CEA, Cellule Carmin, DSV/DIR/CAR et Cab. HC, B.P. 6, 92265 Fontenay-aux-Roses, France.

IRSN, Laboratoire de RadioTOXicologie expérimentale, DRPH/SRBE/LRTOX, B.P. 166, 26702 Pierrelatte, France.
} 


\section{Introduction}

La dose reçue par l'homme suite à une exposition externe n'est pas mesurable. Elle est estimée à partir d'une valeur enregistrée par un dosimètre ou calculée lors de la simulation d'un accident d'irradiation. La dosimétrie d'une exposition interne est plus indirecte encore puisque seules des valeurs d'activité corporelle ou excrétée sont alors mesurées. Celle-ci évolue tant en valeur totale dans le corps qu'en distribution relative entre les différents tissus biologiques avec le temps passé depuis l'incorporation.

Les grandeurs dosimétriques sont communes aux expositions interne et externe (ICRP, 1995 ; JO, 2003a) :

- la dose absorbée $D_{T, R}$ est la quantité d'énergie cédée au tissu $T$ par le rayonnement $R$ et s'exprime en Gray (Gy), avec $1 \mathrm{~Gy}=1 \mathrm{~J} \mathrm{~kg}^{-1}$;

- la dose équivalente $H_{T, R}$ prend en compte l'effet biologique relatif des différents types de rayonnement par l'intermédiaire d'un facteur de pondération $w_{R}$, dont la valeur est recommandée par la Commission internationale de protection radiologique (CIPR) (ICRP, 1990). Elle se calcule, en Sievert (Sv), par la formule : $H_{T}=\sum_{R} w_{R} D_{T, R}$;

- la dose efficace $E$ est une grandeur de radioprotection se rapportant au risque global pour un individu de référence. Elle est calculée, en Sv, comme étant la somme des doses équivalentes reçues par les tissus les plus radiosensibles, pondérée par un facteur $w_{T}$ déduit des études épidémiologiques et biologiques (ICRP, 1990) : $E=\sum_{T} w_{T} H_{T}$.

Le caractère prolongé de l'irradiation due à une contamination interne a conduit à définir le concept spécifique de dose engagée. Celle-ci est définie comme étant la somme des doses équivalentes ou efficaces reçues pendant 50 ans après l'incorporation de radioactivité par un adulte ou jusqu'à l'âge de 70 ans pour un enfant (ICRP, 1995 ; JO, 2003a).

\section{Mesure de la radioactivité}

\subsection{Examen in vivo par anthroporadiamétrie}

L'anthroporadiamétrie est la mesure in vivo des photons émis par les radionucléides présents dans l'ensemble du corps humain ou dans un organe particulier, comme la thyroïde pour l'iode ou les poumons pour les aérosols insolubles. Cette mesure est effectuée à l'aide d'un détecteur généralement constitué d'un cristal scintillateur $\mathrm{NaI}(\mathrm{Tl})$ ou d'un semi-conducteur germanium de 


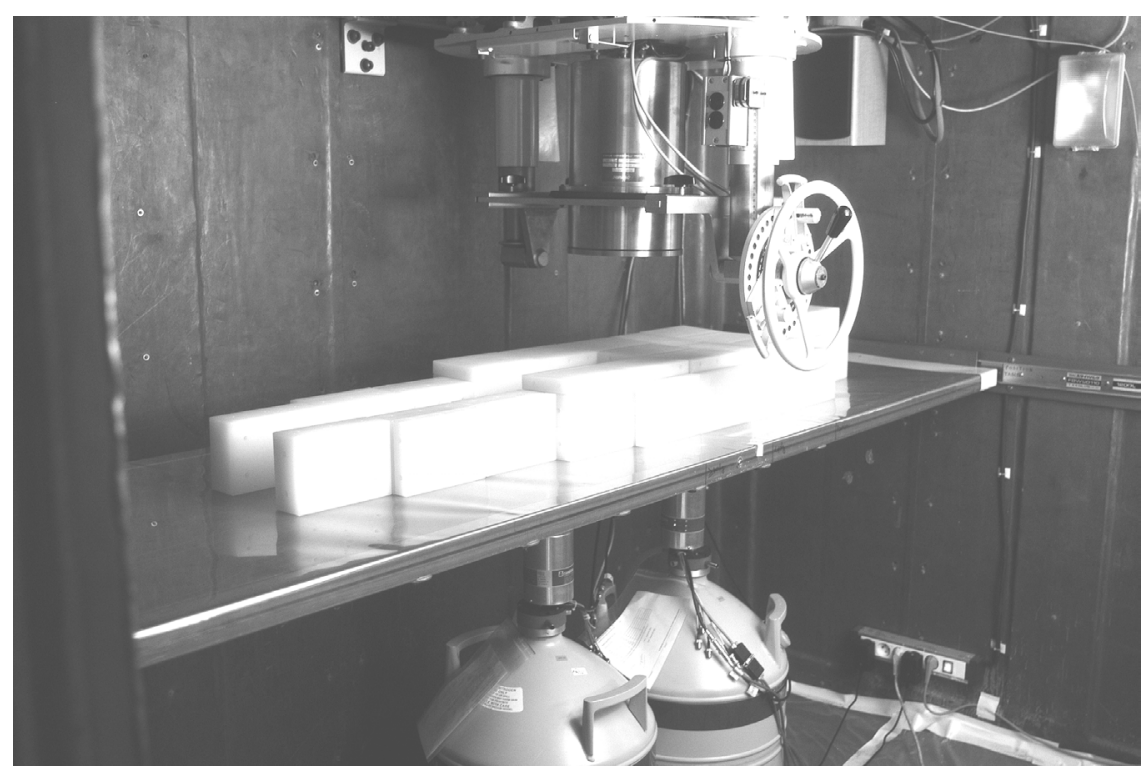

Figure 1 - Système de mesure anthroporadiamétrique haute énergie de l'IRSN à Fontenay-aux-Roses comprenant 1 détecteur NaI (au-dessus) et 2 détecteurs germanium (en dessous). L'installation est présentée avec le fantôme d'étalonnage IGOR, constitué de briques de polyéthylène.

In vivo counting system of IRSN, Fontenay-aux-Roses, for measurement of high energy emitters, including 1 NaI detector (up) and 2 germanium detectors (down). The system is shown with the IGOR calibration phantom made of polyethylene bricks.

haute pureté (GeHP) refroidi à l'azote liquide et placé à l'extérieur du corps (Fig. 1). L'analyse de l'énergie des photons détectés permet ensuite d'évaluer la composition isotopique de la contamination puis de déduire l'activité retenue à partir du taux de comptage et de la comparaison à un étalonnage réalisé sur un mannequin, ou fantôme, anthropomorphe ou à une simulation par un code de calcul Monte Carlo (de Carlan et al., 2005). Un examen anthroporadiamétrique est rapide, couramment de l'ordre de 10 à 30 min, mais n'est possible que pour des radioisotopes émetteurs $X$ ou $\gamma$ d'intensité suffisante et permet d'atteindre des limites de détection de l'ordre de celles indiquées dans le tableau I. Ces limites de détection sont améliorées en protégeant le système de mesure du rayonnement naturel ambiant par un confinement en béton ou en métal.

\subsection{Analyse radiotoxicologique in vitro}

L'analyse radiotoxicologique est la mesure de l'activité excrétée dans les urines ou les selles pendant une période d'échantillonnage, typiquement d'une journée, ou 
TABLEAU I

Limites de détection (LD) in vivo typiques en anthroporadiamétrie de quelques radioisotopes usuels (ICRP, 1997).

Typical detection limit (LD) for in vivo counting of some common radionuclides (ICRP, 1997).

\begin{tabular}{ccc}
\hline radioisotope & tissu cible de la mesure & LD $(\mathrm{Bq})$ \\
\hline${ }^{137} \mathrm{Cs}$ & corps entier & 50 \\
${ }^{60} \mathrm{Co}$ & thyroïde & 50 \\
${ }^{131} \mathrm{I}$ & & 100 \\
${ }^{238} \mathrm{Pu}$ & poumons & 1000 \\
${ }^{241} \mathrm{Am}$ & & 20 \\
${ }^{235} \mathrm{U}$ & & 200 \\
\hline
\end{tabular}

plus rarement de l'activité présente dans un prélèvement sanguin, dans un frottis nasal ou dans une biopsie.

Les radioisotopes émetteurs de photons sont mesurés par spectrométrie $\gamma-X$ similaire à l'anthroporadiamétrie, généralement avec des détecteurs GeHP. Les émetteurs $\beta$ sont détectés à l'aide de compteurs proportionnels à circulation de gaz ou par scintillation liquide. La mesure de l'activité des émetteurs $\alpha$ repose soit sur un comptage de l'activité totale par un compteur proportionnel ou à scintillation, soit sur une spectrométrie $\alpha$ à l'aide de détecteurs semi-conducteurs en silicium précédée d'une purification radiochimique. L'analyse par spectrométrie $\alpha$ permet de déterminer l'isotopie de l'échantillon mais requiert près d'une semaine de minéralisation, de traitement chimique puis de comptage de l'activité. Enfin, la masse des actinides présents dans un échantillon peut être déterminée par les techniques élémentaires que sont l'analyse de la cinétique de phosphorescence (KPA) et la spectrométrie de masse utilisant un plasma à couplage inductif (ICPMS). Très rapide (Bouvier-Capely et al., 2004), sensible et illustrée par la figure 2, cette dernière technique est particulièrement adaptée à la surveillance des isotopes de l'uranium (Baglan et al., 1999). Le tableau II présente des limites de détection typiques obtenues en analyse radiotoxicologique urinaire.

\section{Interprétation dosimétrique des mesures}

\subsection{Modèles biocinétiques}

Les analyses radiotoxicologiques in vitro et les examens anthroporadiamétriques in vivo ne fournissent qu'une information ponctuelle sur l'activité retenue ou excrétée au temps de la mesure. Pour reconstruire l'historique de l'irradiation et en déduire la dose engagée, il est nécessaire de faire une hypothèse sur la biocinétique du radionucléide depuis le moment de l'incorporation jusqu'au terme de la période 


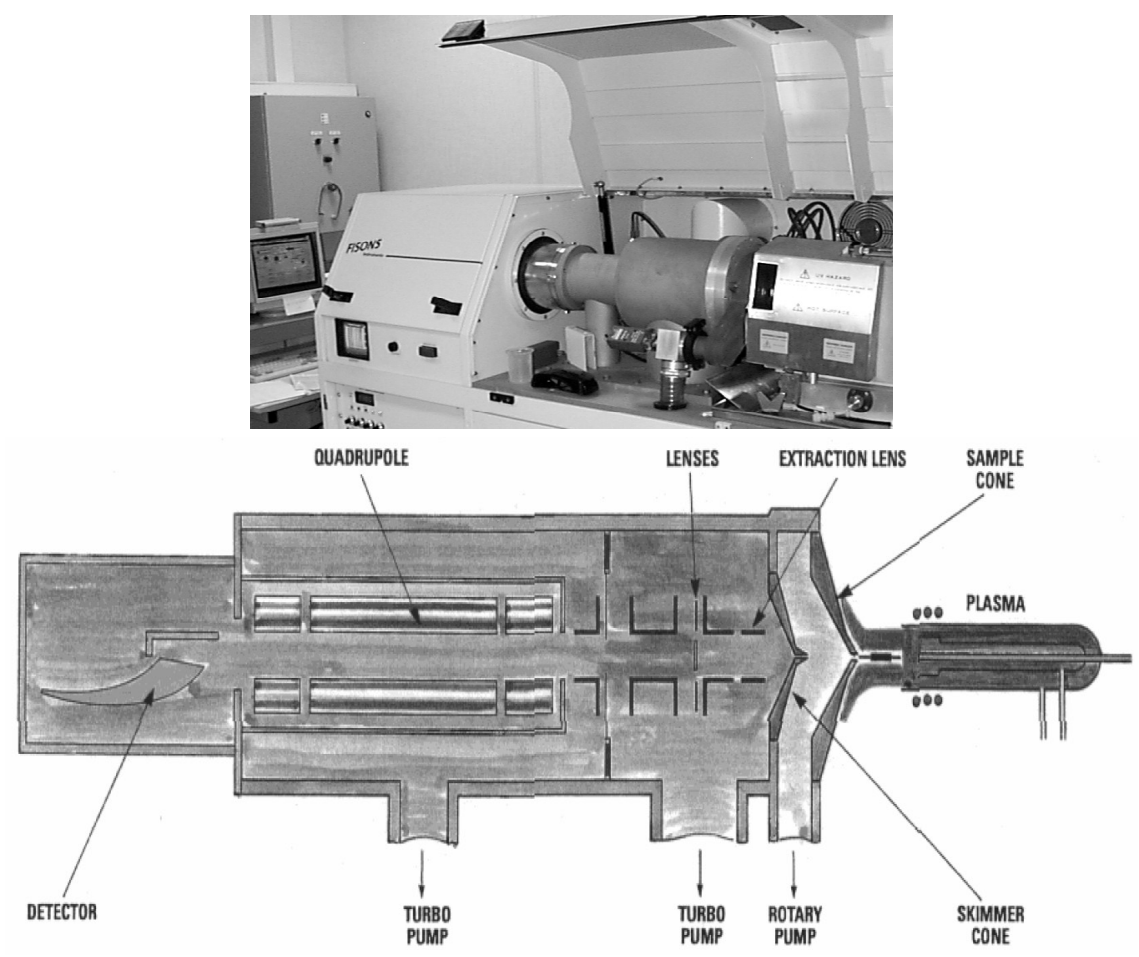

Figure 2 - Photographie et schéma d'un système d'ICP-MS de l'IRSN à Fontenay-aux-Roses. Picture and diagram of an ICP-MS device at IRSN, Fontenay-aux-Roses.

\section{TABLEAU II}

Limites de détection (LD) typiques en analyse radiotoxicologique urinaire (ICRP, 1997 ; Métivier et al., 2006 ; HPA, 2006).

Typical detection limit (LD) for urine analysis (ICRP, 1997 ; Métivier et al., 2006 ; HPA, 2006).

\begin{tabular}{ccc}
\hline radioisotope & technique de mesure & $\mathrm{LD}\left(\mathrm{mBq} \mathrm{L}^{-1}\right)$ \\
\hline${ }^{3} \mathrm{H}$ & scintillation liquide & 100000 \\
${ }^{131} \mathrm{I}$ & spectrométrie $\gamma$ & 1000 \\
${ }^{137} \mathrm{Cs}$ & Purification chimique et spectrométrie $\alpha$ & 30 \\
${ }^{210} \mathrm{Po}$ & $\mathrm{KPA}$ & $25^{(\mathrm{a})}$ \\
$\mathrm{U}$ naturel & Purification chimique et spectrométrie $\alpha$ & 10 \\
$\mathrm{U}$ naturel & ICP-MS & $0,15^{\text {(b) }}$ \\
$\mathrm{U}$ naturel & Purification chimique et spectrométrie $\alpha$ & 1 \\
${ }^{238} \mathrm{Pu}$ & &
\end{tabular}

(a) soit $1 \mu \mathrm{g} \mathrm{L}-1$, (b) soit $5 \mathrm{ng} \mathrm{L}^{-1}$. 


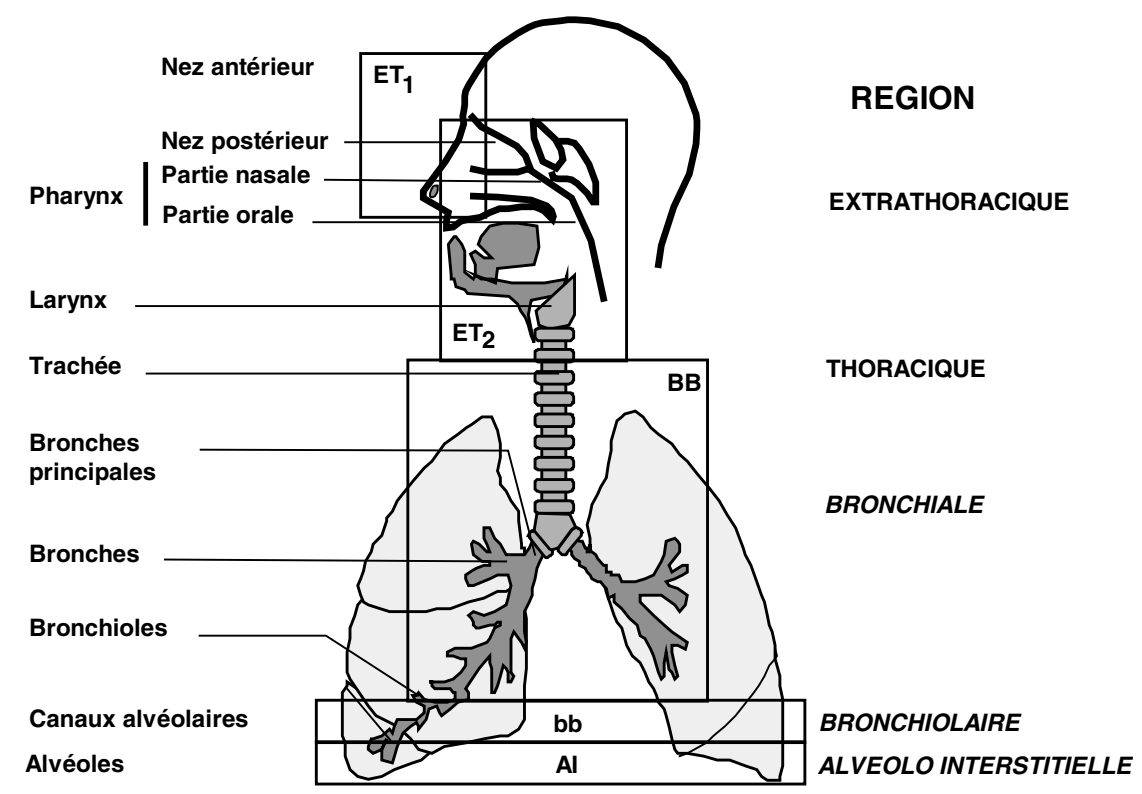

Figure 3 - Régions du modèle respiratoire humain (d'après ICRP, 1994).

Regions of the human respiratory tract model (from ICRP, 1994).

d'engagement de dose. Cette hypothèse se fonde sur l'information collectée par l'expérimentation animale, le suivi de contaminations humaines incidentelles ou volontaires, et synthétisée par la CIPR dans des modèles biocinétiques de référence.

La cinétique de passage d'un radionucléide dans la circulation sanguine dépend de sa voie d'incorporation :

- l'inhalation est le mode de contamination le plus courant pour les travailleurs. Elle est décrite par le modèle respiratoire de la publication 66 de la CIPR (ICRP, 1994) illustré en figure 3. La répartition initiale du dépôt d'activité entre les régions extrathoracique, bronchique, bronchiolaire et alvéolaire des voies respiratoires dépend de la forme physique de l'aérosol contaminant, qui est modélisée en partie par le diamètre aérodynamique médian en activité (DAMA) des particules qui le composent. Ensuite, l'épuration de l'activité déposée se fait simultanément par transport muco-ciliaire vers le tractus gastrointestinal, par transport lymphatique vers les nodules proximaux et par absorption vers la circulation sanguine. La vitesse d'absorption, dépendante de la solubilité du composé chimique, est un paramètre clef du modèle qui peut 


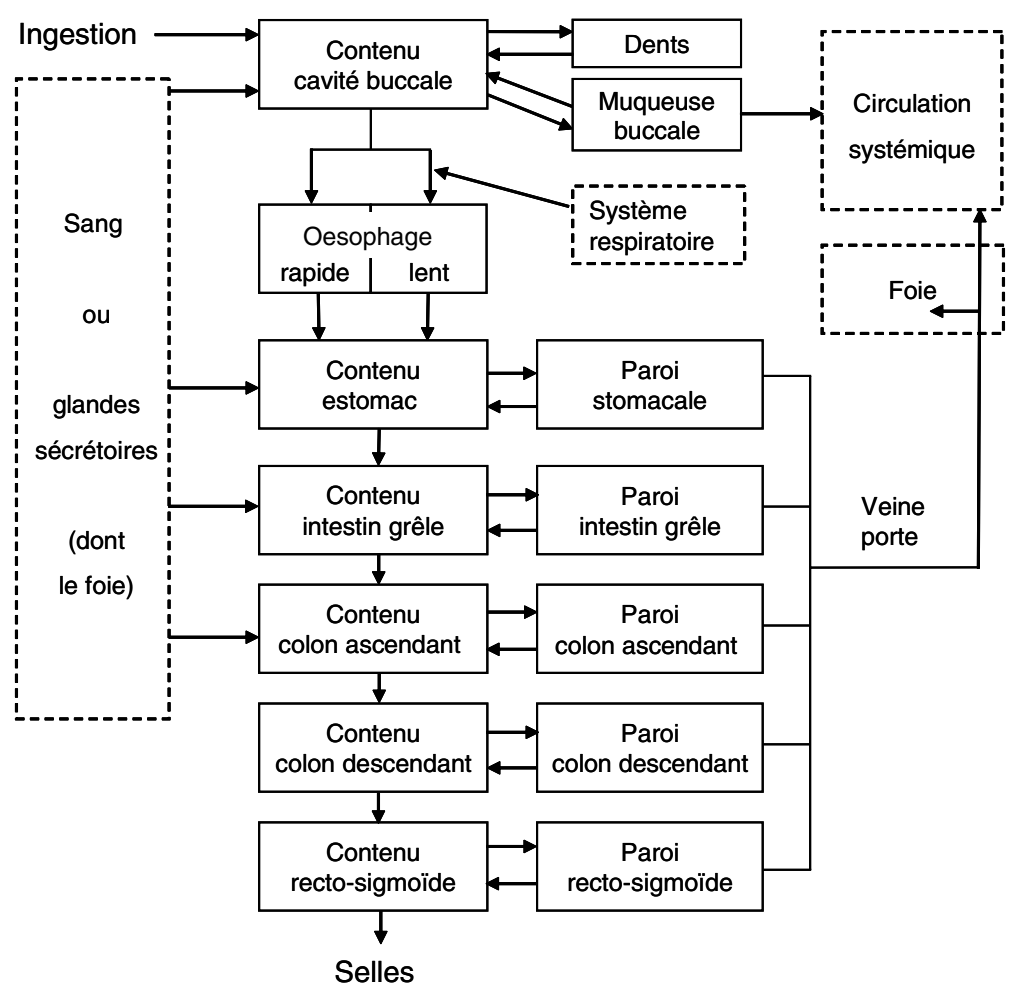

Figure 4 - Structure du modèle alimentaire humain (d'après ICRP, 2006).

Structure of the human alimentary tract model (from ICRP, 2006).

être fixé, à défaut d'information spécifique, suivant trois types d'absorption de référence, rapide $(\mathrm{F})$, modérée $(\mathrm{M})$ ou lente $(\mathrm{S})$;

- le mode de contamination le plus fréquent dans le public est l'ingestion. Le modèle alimentaire de la publication 30 de la CIPR (ICRP, 1979), récemment révisé dans la publication 100 (ICRP, 2006 ; Fig. 4), lui est consacré. L'activité ingérée est transportée successivement de la cavité buccale vers l'œsophage, l'estomac, l'intestin grêle et le gros intestin puis excrétée dans les selles, ou transférée vers la circulation sanguine pour une fraction absorbée $f_{1}$ (ICRP, 1979) ou $f_{A}$ (ICRP, 2006) dépendante de la solubilité du composé chimique ;

- l'injection directe dans la circulation sanguine est normalement réservée au patient de médecine nucléaire. Le transfert cutané à travers la peau saine n'est possible que pour quelques radionucléides dont le tritium. Enfin, si les contaminations par plaie sont rares, elles font l'objet d'une préoccupation 


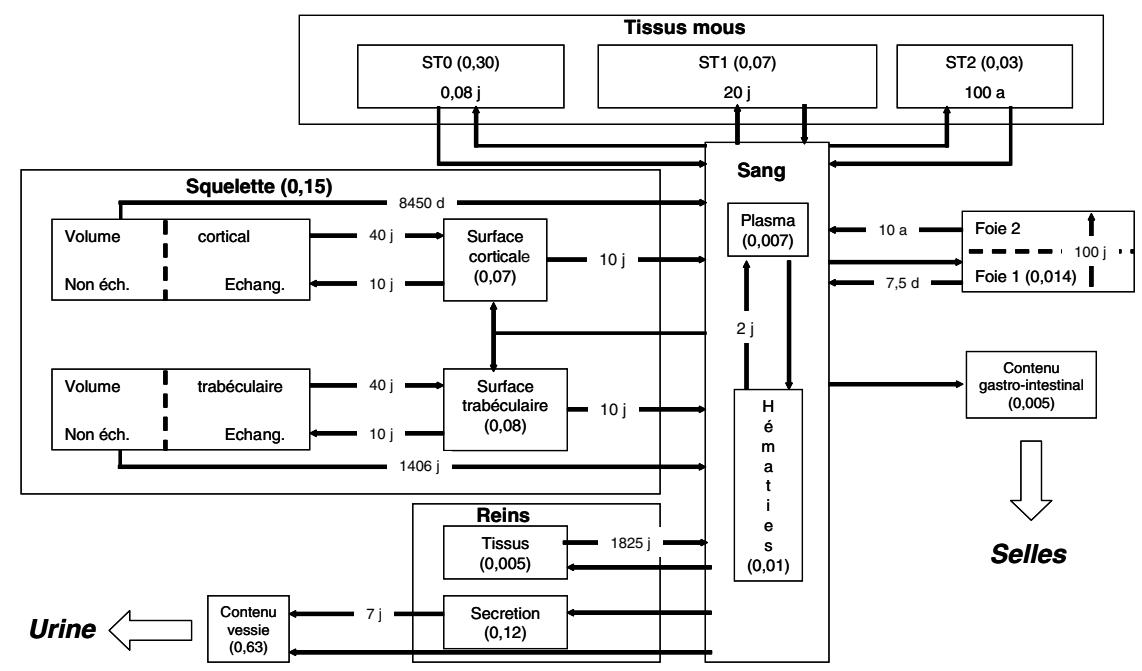

Figure 5 - Structure du modèle systémique de l'uranium (d'après ICRP, 1995). Entre parenthèses sont notées les fractions de l'activité quittant le sang qui entrent dans chaque compartiment. Les durées indiquées correspondent aux périodes de transfert ou de rétention.

Structure of the biokinetic model for uranium (from ICRP, 1995). The fraction of activity leaving blood and entering each compartment is noted in brackets. The indicated times are the transfer and retention periods.

particulière en raison de la complexité de leur prise en charge médicale et des doses élevées qu'elles peuvent induire. Aussi, un modèle spécifique est en cours de développement par le conseil national des États-Unis sur la mesure des radiations et la radioprotection (NCRP), en collaboration avec la CIPR (Breitenstein et al., 2007).

Après son passage dans la circulation sanguine un radionucléide est distribué entre les différents tissus puis retenu ou éliminé par désintégration, excrétion urinaire et fécale. Son tropisme et sa vitesse d'élimination dépendent de l'élément considéré, de sa période radioactive et de sa spéciation chimique. Pour en rendre compte, la CIPR a défini un modèle biocinétique systémique par élément ou groupe d'éléments de propriétés chimiques voisines (ICRP, 1997). À titre d'exemple, la structure du modèle systémique de l'uranium est montrée en figure 5.

Mathématiquement, l'ensemble des modèles biocinétiques de la CIPR correspond à un système linéaire d'équations différentielles du premier ordre à coefficients constants dont la solution $m$ permet de prévoir l'excrétion d'activité et sa rétention dans les organes d'un individu de référence (ICRP, 2002) en fonction du temps écoulé depuis une incorporation unitaire. 

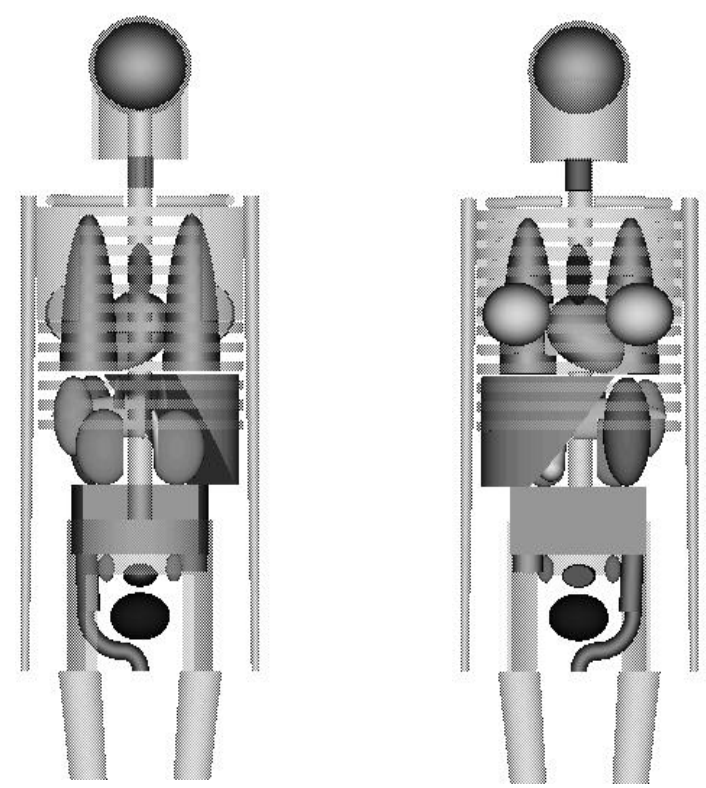

Figure 6-Organes du fantôme représentant l'individu de référence (d'après Cristy et Eckerman, 1993). Gauche, vue de dos. Droite, vue de face. Organs of the phantom representing the reference man (from Cristy and Eckerman, 1993). Left, back. Right, front.

\subsection{Modèles dosimétriques}

Le débit de dose équivalente reçue par un tissu $T$ d'une personne de référence est calculé suivant la formule (ICRP, 1979) :

$$
\dot{H}_{T}=\sum_{S} q_{S} S E E(T \leftarrow S)
$$

où $q_{S}$ est l'activité dans le tissu contaminé $S$ prévue par le modèle biocinétique et $\operatorname{SEE}(T \leftarrow S)$ l'énergie spécifique efficace définie par :

$$
S E E(T \leftarrow S)=\sum_{R} \frac{Y_{R} E_{R} w_{R} A F(T \leftarrow S)_{R}}{m_{T}}
$$

avec $Y_{R}$ la probabilité d'émission de la radiation $R$ par transformation nucléaire du radioisotope, $E_{R}$ l'énergie de $R, w_{R}$ son facteur de pondération, $m_{T}$ la masse de $T$ et $A F(T \leftarrow S)_{R}$ la fraction absorbée dans $T$ de l'énergie émise dans $S$ sous la forme de $R$.

Les fractions absorbées $A F$ sont obtenues en appliquant les équations de transport des radiations dans la matière à un fantôme représentant l'individu de référence (Cristy et Eckerman, 1993), comme celui montré en figure 6. L'intégration sur la période d'engagement puis la sommation pondérée par les 
facteurs $w_{T}$ des doses équivalentes engagées reçues par les tissus permet de calculer un coefficient $e$ de dose efficace engagée par unité d'activité incorporée (DPUI).

\section{Mise en œuvre de la surveillance dosimétrique}

\subsection{Programme de surveillance}

Lorsqu'une exposition par contamination interne due à l'activité humaine est suspectée, une surveillance adaptée doit être conduite pour s'assurer du respect des limites légales de dose efficace de $20 \mathrm{mSv}$ pour les travailleurs exposés et de $1 \mathrm{mSv}$ pour le public (JO, 2003b, 2001). Ces limites s'appliquent au cumul de la dose efficace annuelle reçue par irradiation externe et des doses efficaces engagées consécutivement aux incorporations de l'année. L'ordre de grandeur de l'exposition peut être estimé par la mesure de l'activité d'un prélèvement d'air ambiant ou de la substance contaminée susceptible d'être ingérée mais cette mesure ne permet pas une évaluation dosimétrique précise. Aussi, une surveillance individuelle par anthroporadiamétrie ou analyse radiotoxicologique doit être préférée lorsqu'une exposition significative en regard de la limite de dose est envisageable. Si une contamination interne est possible pendant une période prolongée, un programme de surveillance de routine est mis en place par la réalisation de mesures d'activité à intervalle régulier. Le choix de la technique de mesure et de l'intervalle de surveillance prend en compte les rayonnements émis par le radionucléide, sa vitesse d'élimination par désintégration et excrétion ainsi que la limite de détection. Ainsi, l'Agence internationale à l'énergie atomique recommande que la fréquence de mesure adoptée permette de détecter toute incorporation conduisant à une dose supérieure à $5 \%$ de la limite annuelle (IAEA, 1999). À titre d'exemple, sont présentés dans le tableau III des intervalles de mesure recommandés pour la surveillance de l'exposition professionnelle à quelques radionucléides.

\subsection{Démarche du projet IDEAS}

L'interprétation en termes de dose efficace engagée $E$ d'une mesure d'activité $M, t$ jours après incorporation se fait à l'aide de la fonction de rétention ou d'excrétion $m$ et du coefficient de DPUI $e$, par l'application de la formule : $E=\frac{M}{m(t)} e$.

Cependant, les exercices d'intercomparaison ont montré que la latitude dans le choix du modèle à adopter et la nécessité de faire des hypothèses sur certains des paramètres de l'exposition, comme la date d'incorporation, le DAMA ou le type d'absorption d'un aérosol, lorsqu'ils ne sont pas connus précisément conduit à une 


\section{TABLEAU III}

Intervalles de mesure de surveillance de routine de l'exposition à l'uranium, au plutonium, au césium et à l'iode recommandés par la CIPR (ICRP, 1997) ou le projet européen OMINEX (Etherington et al., 2003). Types d'absorption par défaut : F, rapide ; M, modérée ; $\mathrm{S}$, lente. Intervals for routine monitoring of internal exposure to uranium, plutonium, caesium and iodine as recommended by ICRP (ICRP, 1997) or OMINEX European project (Etherington et al., 2003). Reference types of absorption : F, fast ; M, moderate ; S, slow.

\begin{tabular}{|c|c|c|c|c|c|c|}
\hline contaminant & $\begin{array}{c}\text { type } \\
\text { d'absorption }\end{array}$ & $\begin{array}{l}\text { mesure } \\
\text { directe }\end{array}$ & $\mathrm{LD}(\mathrm{Bq})$ & $\begin{array}{l}\text { mesure } \\
\text { urinaire }\end{array}$ & $\begin{array}{c}\mathrm{LD} \\
(\mathrm{mBq} / 24 \mathrm{~h})\end{array}$ & $\begin{array}{l}\text { mesure } \\
\text { fécale }\end{array}$ \\
\hline $\begin{array}{l}\text { nitrate d'uranium } \\
\text { tributylphosphate } \\
\text { d'uranium } \\
\text { peroxyde d'uranium }\end{array}$ & $\mathrm{F}$ & non & & 30 jours & & non \\
\hline $\begin{array}{c}\text { diuranate } \\
\text { d'ammonium } \\
\text { trioxyde d'uranium } \\
\text { tetrafluorure } \\
\text { d'uranium }\end{array}$ & M & $\begin{array}{l}\text { pulmonaire } \\
180 \text { jours }\end{array}$ & 200 & 90 jours & 0,15 & 180 jours \\
\hline $\begin{array}{l}\text { octoxyde d'uranium } \\
\text { dioxyde d'uranium }\end{array}$ & $S$ & $\begin{array}{l}\text { pulmonaire } \\
180 \text { jours }\end{array}$ & & 90 jours & & 180 jours \\
\hline nitrate de plutonium & M & non & $\begin{array}{c}1000 \\
\text { (ou 20 Bq d'Am-241) }\end{array}$ & 365 jours & 1 & 365 jours \\
\hline $\begin{array}{c}\text { dioxyde de plutonium } \\
\text { MOX }\end{array}$ & $S$ & $\begin{array}{c}\text { forte } \\
\text { exposition }\end{array}$ & & 365 jours & & 365 jours \\
\hline césium-137 & $\mathrm{F}$ & $\begin{array}{l}\text { corps entier } \\
180 \text { jours }\end{array}$ & 50 & 180 jours & 1000 & non \\
\hline iode-131 & $\mathrm{F}$ & $\begin{array}{l}\text { thyroïde } \\
14 \text { jours }\end{array}$ & 100 & 30 jours & 1000 & non \\
\hline
\end{tabular}

grande variabilité dans l'estimation par différents experts de la dose correspondant à un même cas de contamination (Doerfel et al., 2000). Pour palier cette situation, le projet européen IDEAS propose une approche structurée visant à harmoniser la procédure d'estimation de la dose résultant d'une contamination interne (Doerfel et al., 2005, 2006). En cours d'adoption par la CIPR (Stather, 2007), elle distingue 4 niveaux de complexité dans l'évaluation de la dose efficace engagée, à appliquer en fonction de l'ordre de grandeur de dose attendu :

- au niveau 0 , lorsque l'activité mesurée est inférieure à une valeur seuil déterminée par avance suivant l'intervalle de mesure et le modèle biocinétique, 
la dose annuelle est vraisemblablement inférieure au dixième de la limite applicable au public, soit $0,1 \mathrm{mSv}$, et aucune autre évaluation dosimétrique n'est nécessaire ;

- au niveau 1 , pour une dose de l'ordre de 0,1 à $1 \mathrm{mSv}$, une évaluation simple est réalisée en adoptant les valeurs de paramètres recommandées par la CIPR à défaut d'une information spécifique, soit une incorporation supposée au milieu de l'intervalle de surveillance, un DAMA de 1 ou de $5 \mu \mathrm{m}$ et un type d'absorption F, M ou S ;

- au niveau 2, si la dose est susceptible de dépasser la limite applicable au public de $1 \mathrm{mSv}$ (JO, 2001) ou en cas d'incident avéré, il est recommandé de pratiquer plusieurs mesures par différentes techniques et/ou à différents temps. Les dates d'incorporation, DAMA et type d'absorption les plus vraisemblables sont alors recherchés par ajustement de la prévision du modèle aux données de mesure ;

- au niveau 3, si la dose est estimée supérieure à la valeur de $6 \mathrm{mSv}$ définissant une zone contrôlée (JO, 2003b), une évaluation plus sophistiquée est conduite en ajustant l'ensemble des paramètres du modèle jusqu'à obtenir une cohérence satisfaisante entre sa prévision et les données de mesure.

À titre d'illustration, le logigramme de l'une des étapes de l'approche d'IDEAS est montré en figure 7.

\section{Limites de l'exercice}

\subsection{Limites des concepts de dose équivalente et de dose efficace}

Les facteurs de pondération $w_{R}$ et $w_{T}$ à partir desquels sont calculées les doses équivalentes et efficaces (en Sv) se rapportent aux effets stochastiques des radiations ionisantes. Ainsi ces doses ne peuvent être mises en relation qu'avec un risque stochastique d'induction de cancer ou de maladie héréditaire. Le risque d'effets déterministes peut être estimé en revanche à partir de la dose absorbée (en Gy) pondérée par un facteur d'efficacité biologique spécifique de l'irradiation considérée (ICRP, 1989).

De plus, le concept de dose efficace n'est pertinent que dans une démarche de radioprotection. En effet, les coefficients de DPUI se rapportent à un individu de référence (ICRP, 2002) caractérisé par l'anatomie, la physiologie et la radiosensibilité moyennes dont rendent compte les modèles biocinétiques, dosimétriques et facteurs de pondération de la CIPR. La dose efficace calculée au moyen de ces modèles est donc spécifique d'une exposition mais pas de la personne qui la subit. Sa finalité est d'être un outil de gestion prospective du risque. Elle est comparée aux limites réglementaires de dose ou à des objectifs de 


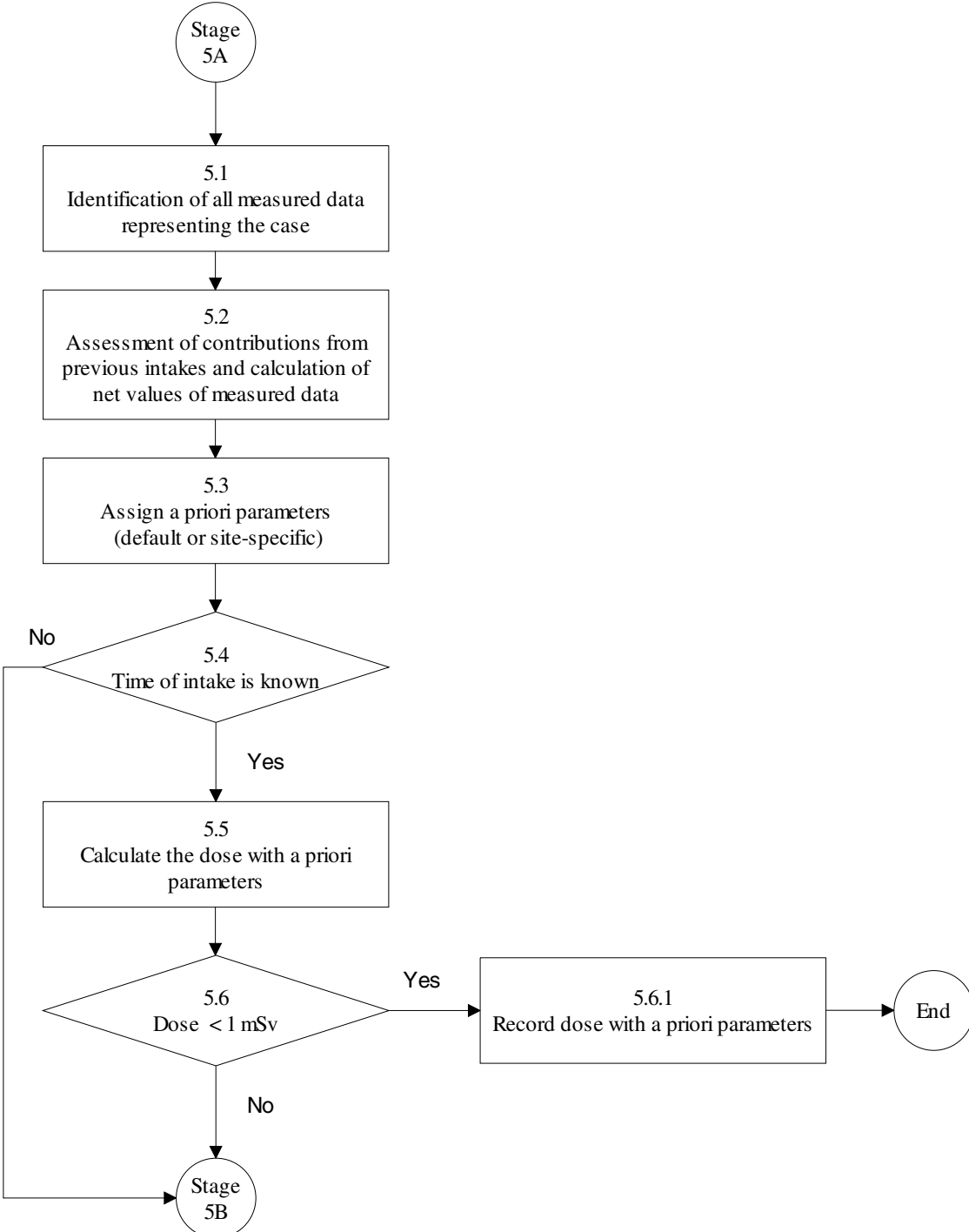

Figure 7 - Logigramme de la procédure IDEAS utilisée pour évaluer la dose au-delà du niveau 1 première partie, évaluation simple à partir de valeurs des paramètres choisies a priori (Doerfel et al., 2006).

Flowchart of the IDEAS procedure for inhalation cases above level 1 - part 1: simple evaluation using parameter values chosen a priori (Doerfel et al., 2006). 
radioprotection supplémentaires afin de détecter ou de prévenir une situation d'exposition jugée anormale et de permettre ainsi la protection de l'ensemble de la population susceptible de subir cette exposition. À cette fin, les limites réglementaires sont fixées à un niveau suffisamment faible pour intégrer les incertitudes liées à la variabilité individuelle (ICRP, 1990). La dose efficace ne doit être utilisée en revanche ni pour une évaluation rétrospective du risque sanitaire individuel ni pour des études épidémiologiques.

\subsection{Incertitude sur le calcul de dose après exposition interne}

L'incertitude sur le risque sanitaire consécutif à une contamination radioactive interne atteint vraisemblablement plusieurs ordres de grandeur (CERRIE, 2004) malgré l'amélioration continue de la sensibilité des techniques de mesure et du réalisme des modèles dosimétriques et épidémiologiques. Toute interprétation d'une valeur de dose en termes de risque doit donc être conduite avec prudence.

En particulier, la précision de l'évaluation dosimétrique est limitée par la sensibilité des techniques de mesure, une activité inférieure à la limite de détection pouvant parfois être associée à une dose significative, et par la fiabilité des modèles dosimétriques. Des lacunes dans la connaissance des suites de certains types d'exposition doivent être comblées par extrapolation à partir d'expositions similaires: de l'expérimentation animale vers le métabolisme humain, d'un élément chimique vers ses analogues, de situations simples comme l'incorporation aiguë vers des scénarios plus complexes comme la contamination chronique (Leggett, 2001). Il s'ensuit que les modèles utilisés comme référence peuvent être plus ou moins réalistes, indépendamment de la variabilité inter et intraindividuelle par rapport à cette référence.

Enfin, comme mentionné au paragraphe 4.2, les intercomparaisons montrent que les modèles et les codes de calcul peuvent être mal utilisés et que des hypothèses plus ou moins pertinentes peuvent être faites en dosimétrie rétrospective lorsque les données d'exposition sont incomplètes.

Ainsi, bien qu'aucune incertitude dépendant de la variabilité individuelle ne soit associée aux coefficients de DPUI par la CIPR, une incertitude significative affecte toute de même l'évaluation de la dose efficace engagée en raison de l'erreur de mesure d'activité, de la connaissance imparfaite des caractéristiques physicochimiques du contaminant et éventuellement de la date d'incorporation. Une large part de la recherche en dosimétrie interne vise à circonscrire ces sources d'incertitude (Etherington et al., 2003). Depuis quelques années, la quantification de l'incertitude sur la dose efficace engagée, voire sur la dose absorbée par les tissus radiosensibles, fait également l'objet de recherches qui s'inscrivent dans la 
démarche d'optimisation en radioprotection (Etherington et al., 2006) et pourraient à long terme contribuer à une estimation de l'incertitude sur le risque individuel.

\subsection{Relation dose-effet}

Il est particulièrement difficile d'établir une relation précise entre la dose estimée suite à une contamination interne et un effet biologique. En effet, les données épidémiologiques permettant de quantifier les effets sanitaires des rayonnements ionisants ne se rapportent qu'à des irradiations d'au moins quelques centaines de mGy. Les effets des doses plus faibles sont donc extrapolés suivant une hypothèse de linéarité sans seuil dont la discussion dépasse l'objet de cet article (ICRP, 2005). Lors d'une exposition interne, la toxicité chimique peut être prépondérante par rapport à la toxicité radiologique, comme c'est le cas pour l'uranium appauvri (IPSN, 2001), ou les deux peuvent interagir en modifiant le métabolisme du radionucléide ou la sensibilité des tissus (Houpert et al., 2005). Enfin, du fait d'un dépôt d'énergie très hétérogène, une dosimétrie réaliste des émetteurs alpha nécessiterait de connaître la distribution cellulaire des radionucléides, la position précise des cellules cibles (Fakir et al., 2005) et les mécanismes de communication inter-cellulaire suivant l'irradiation (Mothersill et Seymour, 2004).

Malgré ces différentes limites, la dose efficace engagée reste un précieux outil de radioprotection mais elle n'a pas été conçue comme un indicateur du risque sanitaire individuel. L'estimation des risques après une contamination radioactive interne est un problème complexe, spécifique à chaque situation, et demeure un sujet de recherche important.

Remerciements. Les auteurs tiennent à remercier Didier Franck pour sa relecture attentive du manuscrit et les illustrations des techniques de mesure de l'activité.

\section{RÉFÉRENCES}

Baglan N., Cossonnet C., Trompier F., Ritt J., Bérard P. (1999) Implementation of ICP-MS protocols for uranium urinary measurements in worker monitoring, Health Phys. 77, 455-461.

Bouvier-Capely C., Ritt J., Baglan N., Cossonnet C. (2004) Potentialities of mass spectrometry (ICPMS) for actinides determination in urine, Appl. Rad. Isotopes 60, 629-633.

Breitenstein B.D., Durbin P.W., Goans R.E., Guilmette R., Russel J.J., Toohey R.E. (2007) The NCRP radionuclide dosimetry models for wounds. NCRP report (à paraître).

CERRIE (2004) Report. Committee Examining Radiation Risks of Internal Emitters, London.

Cristy M., Eckerman K.F. (1993) SEECAL: Program to calculate age-dependent specific effective energies, ORNL/TM-12351. Oak Ridge National Laboratory.

de Carlan L., Roch P., Blanchardon E., Franck D. (2005) New method of voxel phantom creation: application for whole body counting calibration and perspectives in individual internal dose assessment, Radiat. Prot. Dosim. 116, 160-164. 
Doerfel H., Andrasi A., Bailey M.R., Birchall A., Castellani C.-M., Hurtgen C., Jarvis N., Johansson L., Le Guen B., Tarroni G. (2000) Third european intercomparison exercise on internal dose assessment, research report FZKA 6457. Research Center Karlsruhe.

Doerfel H., Andrasi A., Aubineau-Lanièce I., Bailey M., Berkovski V., Blanchardon E., Castellani C.M., Hurtgen C., Jourdain J.-R., Le Guen B., Malatova I., Marsh J., Puncher M. (2005) Guide pratique pour estimer la dose interne à partir des résultats de mesure de surveillance (projet IDEAS), Radioprotection 40, 47-55.

Doerfel H., Andrasi A., Bailey M., Berkovski V., Blanchardon E., Castellani C.-M., Hurtgen C., Le Guen B., Malatova I., Marsh J., Stather J. (2006) General guidelines for the assessment of internal dose from monitoring data (project IDEAS - EU contract $n^{\circ}$ FIKR-CT2001-00160). Report FZKA 7243. Research Center Karlsruhe.

Etherington G., Ansoborlo E., Bérard P., Cossonnet C., Franck D., Genicot J.-L., Hodgson A., Hurtgen C., Jourdain J.-R., Le Guen B., Rahola T., Sovijärvi J., Stradling G.N. (2003) Design and implementation of monitoring programmes for internal exposure (Project OMINEX), Radiat. Prot. Dosim. 105, 641-644.

Etherington G., Birchall A., Puncher M., Molokanov A., Blanchardon E. (2006) Uncertainties in assessments of doses from intakes of radionuclides, Radiat. Prot. Dosim. 121, 40-51.

Fakir H., Hofmann W., Aubineau-Lanièce I. (2005) Microdosimetry of radon progeny alpha particles in bronchial airway bifurcations, Radiat. Prot. Dosim. 117, 382-394.

Houpert P., Lestaevel P., Bussy C,. Paquet F., Gourmelon P. (2005) Enriched but not depleted uranium affects central nervous system in long-term exposed rat, Neurotoxicology 26, 1015-1020.

HPA (2006) Method for the assessment of polonium-210 in urine being used by HPA for the UK Po210 incident. HPA, Chilton.

IAEA (1999) Assessment of occupational exposure due to intakes of radionuclides, safety standards series $R S-G$-1.2. IAEA, Vienna.

ICRP Publication 30 (1979) Part 1: Limits for intakes of radionuclides by workers, Ann. ICRP 2 (3/4).

ICRP Publication 58 (1989) RBE for deterministic effects, Ann. ICRP 20 (4).

ICRP Publication 60 (1990) 1990 Recommendations of the international commission on radiological protection, Ann. ICRP 21 (1-3).

ICRP Publication 66 (1994) Human respiratory tract model for radiological protection, Ann. ICRP 24 (1-3).

ICRP Publication 69 (1995) Age-dependent doses to members of the public from intakes of radionuclides: Part 3. Ingestion dose coefficients, Ann. ICRP 25 (1).

ICRP Publication 78 (1997) Individual monitoring for internal exposure of workers. Replacement of ICRP Publication 54, Ann. ICRP 27 (3-4).

ICRP Publication 89 (2002) Basic anatomical and physiological data for use in radiological protection: reference values, Ann. ICRP 32 (3-4).

ICRP Publication 99 (2005) Low-dose extrapolation of radiation-related cancer risk, Ann. ICRP 35 (4).

ICRP Publication 100 (2006) Human alimentary tract model for radiological protection, Ann. ICRP 36 $(1-2)$.

IPSN (2001) État des connaissances sur les risques potentiels associés à l'uranium appauvri utilisé dans les armes, Rapport DPHD/2001-01. IPSN, Fontenay-aux-Roses.

JO (2001) Décret $n^{\circ} 2001-215$ du 8 mars 2001 modifiant le décret $n^{\circ} 66-450$ du 20 juin 1966 relatif aux principes généraux de protection contre les rayonnements ionisants (J.O. n ${ }^{\circ} 59$ du 10 mars 2001 , p. 3869).

JO (2003a) Arrêté du $1^{\text {er }}$ septembre 2003 définissant les modalités de calcul des doses efficaces et des doses équivalentes résultant de l'exposition des personnes aux rayonnements ionisants (J.O. $\mathrm{n}^{\circ} 262 \mathrm{du} 13$ novembre 2003, p. 19326). 
JO (2003b) Décret $n^{\circ}$ 2003-293 du 31 mars 2003 relatif à la protection des travailleurs contre les dangers des rayonnements ionisant (J.O. $n^{\circ} 78$ du 2 avril 2003, p. 5779).

Leggett R.W. (2001) Reliability of the ICRP's dose coefficients for members of the public. I. Sources of uncertainty in the biokinetic models, Radiat. Prot. Dosim. 95, 199-213.

Métivier H., Aubineau-Lanièce I., Blanchardon E., Bouvier-Capely C., de Carlan L., Franck D., Paquet F. (2006) Dosimétrie et surveillance de l'exposition interne, dans Radioprotection et ingénierie nucléaire (H. Métivier, Ed.), pp. 145-176. EDP Sciences, Les Ulis.

Mothersill C., Seymour C.B. (2004) Radiation-induced bystander effects - Implications for cancer, Nature Rev. Cancer 4, 158-164.

Stather J.W. (2007) The work of committee 2 of ICRP on internal dosimetry. Dans: Internal dosimetry of radionuclides, 2-5 October 2006, Montpellier, à paraître dans Radiat. Prot. Dosim. Oxford university press. 